\section{La Organización Mundial de la Salud cumple 50 años}

El 7 de abril de 1998 cumplió 50 años de existencia la Organización Mundial de la Salud. Durante ese medio siglo, la longevidad humana casi se ha doblado; es decir, la esperanza de vida al nacer ha aumentado de 46 años a principios de los años cincuenta a casi 66 años en 1996. Este logro sensacional se debe en gran medida al extenso trabajo que ha realizado la OMS en todo el mundo por conducto de sus seis oficinas regionales: la de África, ubicada en Brazzaville, Congo; la del Mediterráneo Oriental, en Alejandría, Egipto; la de Europa, en Copenhague, Dinamarca; la del Asia Sudoriental, en Nueva Delhi, India; la del Pacífico Occidental, en Manila, Filipinas; y la de las Américas (Oficina Sanitaria Panamericana y Organización Panamericana de la Salud), en Washington, D.C. Otros grandes éxitos han sido la erradicación de la viruela y la erradicación de la poliomielitis en todo el continente americano, victoria próxima a extenderse al resto del mundo. Menos espectaculares quizá, pero no menos importantes, han sido todos los avances conseguidos en la prevención y el control de muchas otras enfermedades, la diseminación de estándares y normas, la concienciación del mundo en cuanto a saneamiento y cuidado del ambiente, el desarrollo de recursos humanos locales y la propagación de conceptos universales relacionados con la salud.

Los fundadores de la OMS fueron visionarios que plasmaron en la Constitución de la Organización la idea de la salud como derecho humano. El lenguaje que aparece en la Constitución de la OMS es aun más liberal e inclusivo (véase la página 284) que el de la Declaración Universal de Derechos del Hombre, la cual acaba de llegar también a su cincuentenario. Hasta la fecha solo hay un documento internacional de derechos humanos que realmente proclama el derecho a la salud: el Protocolo de San Salvador, aprobado en 1988 en el seno de la Organización de los Estados Americanos. Sin embargo, hay varios otros instrumentos internacionales que abordan la salud y cuestiones afines. Entre los más importantes destacan el Convenio Internacional sobre Derechos Económicos, Sociales y Culturales, de 1966; la Convención para la Eliminación de Todas las Formas de Discriminación contra la Mujer, de 1979; y la Convención sobre los Derechos del Niño, de 1989. La OMS desempeña una función notable en la ejecución de todos los artículos que tienen que ver con la salud. Así, trabaja estrechamente con la Oficina del Alto Comisionado para los 
Derechos Humanos, con los comités que monitorean los informes de partidos estatales a los instrumentos mencionados y con sus propios Estados Miembros a los cuales brinda cooperación para convertir las declaraciones en realidad.

La OMS participa en la Comisión de Derechos Humanos y la Subcomisión para la Prevención de Discriminación y Protección de Minorías. En 1997 la Comisión se ocupó de diversos asuntos que tienen consecuencias directas e indirectas para la salud: los derechos humanos y la pobreza extrema, los derechos humanos y el ambiente; los efectos adversos del movimiento ilícito y dumping de desechos tóxicos y otros productos peligrosos; la violencia contra la mujer; los derechos humanos y la bioética; las prácticas tradicionales que afectan a la salud de mujeres y niños; los derechos humanos y VIH/sida; los derechos humanos y los éxodos en masa; las personas desplazadas en sus países; los derechos humanos de personas con discapacidades; alegaciones de la extirpación ilegal de órganos y tejidos en niños; y los pueblos indígenas.

Además, la OMS trabaja en las conferencias de ámbito mundial que auspician las Naciones Unidas. Su presencia sobresalió en la Conferencia Mundial sobre Derechos Humanos de 1993, primer foro mundial en el que se declaró abiertamente que los derechos de la mujer son derechos humanos. Debido en parte a los esfuerzos de la OMS a favor de ese principio, la Declaración y el Programa de Acción de Viena proporcionan marcos nacionales e internacionales para poner en operación el concepto. A pesar de su creciente importancia, el derecho a la salud aún no ha llegado a recibir la atención que merece para poder entrar en la corriente principal de promoción y protección de otros derechos humanos.

\section{Precursores de la OMS}

El primer intento de crear un mecanismo internacional de salud pública para combatir las epidemias de enfermedades infecciosas se remonta a 1851. Ese año, como consecuencia del brote de cólera que en 1830 había diezmado la población europea, se celebró en París la primera Conferencia Sanitaria Internacional con objeto de crear una convención del mismo nombre. Esta no se llegó a materializar hasta 1892, cuando después de muchos esfuerzos, surgió la primera Convención Sanitaria Internacional cuya acción estaba limitada al cólera. Cinco años más tarde se adoptó una convención similar sobre la peste.

En 1902 se creó en Washington, D.C., la Oficina Sanitaria Internacional que con el tiempo se convertiría en la actual Organización Panamericana de la Salud. En 1907, se estableció en París la Office Internationale d'Hygiène Publique (OIHP), con una secretaría permanente y un comité de funcionarios de salud pública de 12 Estados Miembros, nueve de los cuales eran europeos. La Liga de las Naciones se creó en 1919 con la tarea de intervenir en cuestiones internacionales de prevención y control de enfermedades y la Organización de Salud de la Liga de las Naciones se radicó en Ginebra, paralela a la OIHP.

Al correr del siglo, entre otros hitos de la salud pública internacional se destacaron la revisión de 1926 de la Convención Sanitaria Internacional, cuando se incluyeron provisiones contra la viruela y el tifo, y la adopción en 1935 de la Convención Sanitaria Internacional para la navegación aérea. En 1938 se celebró en París la última Conferencia Sanitaria Internacional y, ese mismo año, un consejo de salud internacional situado en Alejandría pasó a manos del gobierno de Egipto. Así este consejo, que había sido uno de cuatro organismos de salud regionales instituidos en el siglo XIX para regular los procedimientos de cuarentena en la zona del Mediterráneo, se convirtió en el Consejo Sanitario, Marítimo y de Cuarentena de Egipto. Sin embargo, en 1946 el Consejo volvió a adquirir carácter regional y todas sus funciones se transfirieron en 1949 a la ya existente Organización Mundial de la Salud.

La Conferencia de las Naciones Unidas sobre Organizaciones Internacionales, al reunirse en San Francisco en 1945, aprobó por unanimidad la propuesta del Brasil y China de que se estableciera una nueva organización autónoma de salud internacional. En 1946 la Conferencia Sanitaria Internacional se reunió en Nueva York, en cuya ocasión se aprobó la Constitución de la Organización Mundial de la Salud y se estableció una comisión interina de la OMS. Esta comenzó sus tareas muy pronto, pues al año siguiente fue necesario prestar ayuda a Egipto para combatir una epidemia de cólera. El 7 de abril de 1948 entró en vigencia la Constitución de la OMS con 61 Estados firmantes. Desde entonces esa fecha se conmemora anualmente como Día Mundial de la Salud.

\section{Fundamentos conceptuales de la OMS}

La OMS nació como respuesta al deseo milenario de la humanidad de liberarse de las enfermedades y disfrutar de una larga vida plena de salud. Como es bien sabido, la primera declaración que aparece en la Constitución de la OMS reza: "La salud es un estado de completo bienestar físico, mental y social, y no solamente la ausencia de afecciones o enfermedades". En ese mismo documento 
se definen una serie de principios menos citados pero de suma importancia como fundamentos para la felicidad, las relaciones armoniosas y la seguridad de todos los pueblos del mundo.

- El goce del grado máximo de salud es uno de los derechos fundamentales de todo ser humano sin distinción de raza, religión, ideología política o condición económica o social.

- La salud de todos los pueblos es una condición fundamental para lograr la paz y la seguridad, y depende de la más amplia cooperación de las personas y de los Estados.

- Los resultados alcanzados por cada Estado en el fomento y protección de la salud son valiosos para todos.

- La desigualdad de los diversos países en lo relativo al fomento de la salud y el control de las enfermedades, sobre todo las transmisibles, constituye un peligro común.

- El desarrollo saludable del niño es de importancia fundamental; la capacidad de vivir en armonía en un mundo que cambia constantemente es indispensable para este desarrollo.

- La extensión a todos los pueblos de los beneficios de los conocimientos médicos, psicológicos y afines es esencial para alcanzar el más alto grado de salud.

- Una opinión pública bien informada y una cooperación activa por parte del público son de importancia capital para el mejoramiento de la salud del pueblo.

- Los gobiernos tienen responsabilidad en la salud de sus pueblos, la cual sólo puede ser cumplida mediante la adopción de medidas sanitarias y sociales adecuadas.

Estas proclamaciones son eco de las aspiraciones de los Estados Miembros en la época posguerra de los años cuarenta. A medida que la OMS fue adquiriendo experiencia en diversos lugares del mundo, se fue manifestando cada vez más claramente la necesidad de llevar esa visión a la práctica mediante la creación de un marco conceptual, estrategias y principios operacionales. El marco conceptual se hizo realidad en 1977, cuando la $30^{\mathrm{a}}$ Asamblea Mundial de la Salud decidió que la principal meta social de los gobiernos y de la OMS en los decenios siguientes debería consistir en "alcanzar para todos los ciudadanos del mundo en el año 2000 un grado de salud que les permit[ier]a llevar una vida social y económicamente productiva". El resto es parte de la historia de la salud pública. En la Conferencia Internacional sobre la Atención Primaria de la Salud celebrada en Alma-Ata en 1978, se hizo hincapié en que la atención primaria sería la estrategia central para el logro de la meta de salud para todos en el año 2000, "como parte del desarrollo general conforme al espíritu de justicia social". En aquel tiempo la visión de la meta incluía elementos que todavía no se han cumplido para todos, aun en nuestros días tan cercanos al año 2000: educación sanitaria para todos en métodos de prevención y control de las enfermedades endémicas, promoción de la buena nutrición, suministro suficiente de alimentos y agua salubre, saneamiento básico, atención de salud materna e infantil incluida la planificación familiar, inmunización contra las infecciones principales, prevención y control de las enfermedades y lesiones más comunes y provisión de medicamentos esenciales.

\section{La meta de salud para todos}

Al lanzar la estrategia de atención primaria, se esperaba que los países en general tuvieran una interpretación similar de que "salud para todos" significaba acceso a ese primer escalón y desde allí a todos los niveles de los sistemas de salud con el objetivo siempre presente de mejorar constantemente la salud de toda la población. No obstante, pronto se hizo evidente que el concepto y la interpretación práctica de la meta proclamada por todos variaba tanto de un país a otro como entre grupos de países según sus características sociales y económicas, el estado de salud y los patrones de morbilidad de las poblaciones, y el grado de desarrollo de sus sistemas de salud. El Consejo Ejecutivo de la OMS señaló que los problemas podían abordarse de diferentes formas. Mientras que algunos países tenían la capacidad para entregar la gama completa de servicios, empezando con las personas más necesitadas hasta llegar a toda la población, otros al principio solo podrían ofrecer cierto número de servicios y ampliarlos progresivamente. También se subrayó la realidad de que el planeamiento y la ejecución de las estrategias era un proceso de largo plazo que había que extender poco a poco tanto desde el punto de vista geográfico como de todos los componentes esenciales. Aun más importante, el sector de la salud por sí solo no podía lograr un grado de salud aceptable para todos los ciudadanos de un país; además de la voluntad política y el compromiso de cada gobierno, se necesitaría una coordinación de esfuerzos de todos los sectores de la sociedad. La meta era, pues, una llamada a la justicia social y una estrategia hacia el mejoramiento paulatino de la salud.

\section{Los últimos 20 años}

En los últimos dos decenios, el mundo ha aceptado cada vez más el marco conceptual de 
salud para todos, si bien pasó bastante tiempo antes de que los recursos humanos y financieros apropiados se reorientaran para apuntalar las bases de la atención primaria. Teniendo en cuenta las grandes variaciones entre naciones y poblaciones, puede decirse que la atención primaria, junto con los avances económicos, educacionales y tecnológicos, ha contribuido en muchos países a ampliar las infraestructuras de salud, cultivar la alfabetización, aumentar los ingresos y mejorar la nutrición, el saneamiento, la educación y las oportunidades, especialmente para las mujeres.

Las enfermedades infecciosas han disminuido y la viruela se ha erradicado en todo el mundo. La poliomielitis está también próxima a erradicarse, mientras que la prevención y el control de otras enfermedades como el sarampión y la difteria han reducido la mortalidad y morbilidad infantiles. Millones de niños sobreviven hasta la edad adulta gracias a intervenciones tempranas de salud. Sin duda, el logro más importante de la salud pública en los últimos 50 años ha sido el de alargar la vida humana. Además, la brecha entre la esperanza de vida en los países ricos y en los pobres se ha reducido de 25 años en 1955 a 13,3 años en 1995. Aun así, el progreso hacia la meta de salud para todos se ha visto obstaculizado por un desarrollo socioeconómico muy lento en algunos países, falta de compromiso político en otros, mal uso de recursos y costo elevado de las tecnologías, poca cooperación intersectorial, apoyo insuficiente a la formación de recursos humanos, y diversos elementos entre los que destacan las inaceptables circunstancias sociales y económicas de las mujeres.

Durante los años ochenta, la crisis de la deuda externa hizo que muchos países redujeran los gastos en servicios sociales y de salud. En los noventa, cambios políticos acompañados de disturbios civiles han perjudicado el desarrollo económico y sanitario. En algunos países, sobre todo los recientemente independizados, hasta se perdió lo ganado anteriormente en años de vida y normas de salubridad. La salud ha sufrido los mayores deterioros allí donde las economías no pueden garantizar ingresos adecuados para todos, los sistemas sociales se han colapsado o los recursos ambientales han sido inadecuados o mal administrados. La pobreza y el aumento de las inequidades sociales también han impedido en gran medida el progreso en salud. Hay casi 1300 millones de personas que viven en suma pobreza, una de las causas primordiales de desnutrición y enfermedad. En las naciones más pobres, la falta de fondos para los servicios sociales y de salud y el fracaso de los gobiernos en conseguir fondos internacionales son serios impedimentos para el progreso a la meta. En los lugares que no han podido mantener los servicios básicos, la salud de la población se ha deteriorado, especialmente frente a la nueva amenaza de las enfermedades nuevas o reemergentes. Esto ha dado lugar al crecimiento de los servicios privados en las naciones de ingresos medianos, pero no se han forjado asociaciones efectivas entre los proveedores de servicios públicos y privados.

Para responder a los cambios socioeconómicos, tecnológicos y epidemiológicos que matizan la nueva realidad mundial, en mayo de 1995 la Asamblea Mundial de la Salud pidió que se renovara la estrategia de salud para todos. En consecuencia, se ha desarrollado una nueva política de salud holística basada en la equidad y la solidaridad, con énfasis en que las personas, las familias y la comunidad se hagan responsables de su salud y la sitúen en el marco general del desarrollo. "Salud para todos en el siglo XXI" se ha ido estructurando sobre los logros de la meta original y servirá de guía para actividades y políticas de salud internacionales, regionales, nacionales y locales. La meta renovada ha de ser, sobre todo, el producto de un proceso de consulta muy extenso e inclusivo entre la OMS y los Estados.

\section{La nueva Directora General de la OMS}

En mayo de 1998 ascendió al puesto de Directora General de la OMS la doctora Gro Harlem Brundtland, médica natural de Noruega y ex primera ministra de ese país. Desde muy joven la doctora Brundtland mostró afición por que la ciencia se tuviera más en cuenta en el ámbito político y opina que el ramo de la medicina más cercano a la política es la salud pública, disciplina que estudió en la Universidad de Harvard. La Directora General se especializó en medicina preventiva en Oslo y en sus estudios de posgrado en los Estados Unidos se concentró en la contaminación del medio y otros problemas ambientales. Al volver a su país, trabajó en el sector nacional de la salud maternoinfantil y fue miembro del Consejo de Sanidad de Oslo.

En 1974 la doctora Brundtland fue asignada a dirigir un nuevo Ministerio de Ambiente para su país, en el cual estableció un programa para crear reservas naturales. Luego fue elegida al parlamento por el partido laborista y en 1981, a la edad de 41 años, primera ministra. Fue así la mujer más joven del mundo en encabezar un país occidental, mientras que ahora es la primera mujer en dirigir el destino de la OMS. Como presidenta de la Comisión Mundial sobre el Ambiente y el Desarrollo, de 1984 a 1987, ella promovió el concepto de un desarrollo que satisfaga las necesidades del presente sin comprometer la capacidad de las futuras generaciones de satisfacer las suyas. 
Habiendo manejado, durante sus 10 años como primera ministra, un presupuesto 70 veces mayor que el de la OMS, la doctora Brundtland sabe cómo asignar recursos y establecer prioridades políticas. La Fundación Rockefeller le aportará un fondo de US\$ 2,5 millones para que pueda contratar expertos de alto nivel de fuera de la OMS, que funcionen como asesores durante el próximo período de transición de uno a dos años. Así estará en libertad para escoger entre las personas más talentosas del mundo.

Hace dos meses, la Directora General anunció a todo el personal de la sede y de las oficinas regionales que había creado un nuevo sistema de gabinete para la OMS, con nueve altos funcionarios de todas partes del mundo ya nombrados directores ejecutivos de las áreas técnicas de salud más importantes de nuestros días. Esas áreas incluyen desarrollo y medio ambiente, salud de la familia, tecnología para la salud, políticas de medicamentos, efectos de los cambios sociales en la salud mental, enfermedades transmisibles, enfermedades no transmisibles, fundamentos e información para la política, relaciones externas y cuerpos directivos, y administración general. La oficina de la Directora General contará también con cuatro asesores políticos de mucha experiencia. Ella desea una estructura interna menos vertical, mejor comunicación entre todas las dependencias de la OMS, mayor transparencia y una distribución más clara de responsabilidades. No aprueba de una cultura organizacional regida por la burocracia, sino de una que promueva el desempeño y los resultados. Externamente, se propone un acercamiento al resto de la familia del sistema de las Naciones Unidas para unir fuerzas en pro de la salud, el desarrollo y la reducción de la pobreza.

Como primer acto oficial, la doctora Brundtland puso en ejecución un nuevo código de con- ducta para el personal de alto nivel de la OMS. Todos están obligados a revelar la posesión de valores financieros y a deshacerse de los que puedan constituir un conflicto de intereses. En lo que se refiere a nuevos temas de trabajo, bajo su dirección se han iniciado dos proyectos, uno sobre la malaria y otro antitabaco. Otra prioridad es la lucha contra la epidemia de infección por VIH y sida, pues la situación ha empeorado más de lo anticipado a principios de los años noventa y se ha convertido en un grave obstáculo para el desarrollo.

A la Directora General nada en la vida le parece más importante que servir y marcar una diferencia ayudando a edificar comunidades saludables y combatiendo la enfermedad, la pobreza y el sufrimiento. Sus dotes políticas la hacen una idónea abogada de la salud en el ámbito mundial.

\section{SYNOPSIS}

\section{The World Health Organization reaches its 50th year}

In celebration of the World Health Organization's 50th anniversary, this article features WHO's contribution to the world by examining its current activities in the areas of health, human rights, and development. It briefly summarizes events leading to its establishment over the period from 1851 to 1948, which marks the year when WHO assumed its role as a specialized body of the United Nations. Quoting from various articles in WHO's Constitution, it illustrates the principles that prompt its actions and that have led nations to become aware of their potential goals, thus steering them toward a brighter future. A brief overview of the last 20 years ends with the introduction of WHO's new Director General, Dr. Gro Harlem Brundtland. 